\title{
GLOBAL CONVEXITY PROPERTIES OF SOME FAMILIES OF THREE-DIMENSIONAL COMPACT LEVI-FLAT HYPERSURFACES
}

\author{
DAVID E. BARRETT
}

\begin{abstract}
We consider various examples of compact Levi-flat hypersurfaces in two-dimensional complex manifolds, exploring the interplay between geometric properties of the induced foliation, behavior of the tangential CauchyRiemann equations along the hypersurface, and pseudoconvexity properties of small neighborhoods of the hypersurface.
\end{abstract}

A real hypersurface $S$ of class $C^{2}$ in a complex manifold $M$ is said to be Levi-flat if its Levi-form vanishes identically. By a theorem of Frobenius this is equivalent to saying that $S$ admits a codimension-one foliation $\mathscr{F}$ by complex hypersurfaces of $M$. (The foliation $\mathscr{F}$ is uniquely determined, for the tangent space to $\mathscr{F}$ at $p \in M$ must coincide with the maximal complex subspace of $T_{p} M$.)

In this paper we are interested in exploring the interplay between the geometric properties of $\mathscr{F}$, the pseudoconvexity properties of $M \backslash S$, and the behavior of the tangential Cauchy-Riemann equations along $S$. (In our case the tangential Cauchy-Riemann equations are just the usual Cauchy-Riemann equations along leaves of $\mathscr{F}$.) We will interest ourselves particularly in the case where $S$ is compact and $\operatorname{dim}_{\mathbb{C}} M=2$. The former restriction guarantees that $M$ does not admit a strictly plurisubharmonic function, for no such function admits a maximum at any point of $S$; in particular, $M \neq \mathbb{C}^{n}$. The latter restriction implies that the leaves of $\mathscr{F}$ have complex dimension one; it seems clear that one should expect the higher-dimensional theory to have a somewhat different flavor (see in particular the example of [DF]).

We will also assume in general that $S$ is orientable and connected, and it will occasionally be convenient to shrink $M$ freely, assuming in particular that $S$ separates $M$ into two components.

We take as a starting place Ohsawa's example of a compact Levi-flat hypersurface occurring as a boundary of the product of an annulus with the punctured plane $\mathbb{C}^{*}[\mathrm{Oh}]$. This paper is largely devoted to the study of various classes of compact Levi-flat hypersurfaces extending Ohsawa's example.

In $\S 3$ we study a class of $\mathbb{T}^{2}$-invariant CR structures on $\mathbb{T}^{3}$. The global function-theoretic, pseudoconvexity, and foliation-theoretic properties of structures in this class are very nicely correlated, as explained in the statement of Theorem 1. In $\S 4$ a similar correlation is exhibited for the boundaries of disc

Received by the editors May 16, 1990 and, in revised form, June 6, 1990.

1980 Mathematics Subject Classification (1985 Revision). Primary 32F25.

Supported in part by a grant from the NSF. 
bundles over compact Riemann surfaces. In $\S 5$ use some ideas from dynamics to construct some CR structures which do not fit neatly into the dichotomies of the preceding two sections. $\S \S 1$ and 2 contain some preliminary remarks about the tangential CR equations and straightening of Levi-flat hypersurfaces.

In the course of proving Theorem 1 we construct an example of a smooth bounded domain $D$ in a complex manifold such that holomorphic functions on $D$ cannot in general be approximated uniformly on compact subsets by holomorphic functions which grow like a negative power of the distance to the boundary.

\section{Remarks about the tangential CR EQUATIONS}

The complex vector fields on a complex manifold $M$ which annihilate local holomorphic functions are known as vector fields of type $(0,1)$. A CR function $f$ on a open subset of a real hypersurface $S$ in $M$ is a function which is annihilated by all vector fields of type $(0,1)$ which are tangent to $S$; CR distributions and hyperfunctions are defined similarly. If $S$ is Levi-flat then a CR function is simply a function which is holomorphic along leaves of $\mathscr{F}$. A function $f$ on $S$ is constant on leaves of $\mathscr{F}$ if and only if $f$ is annihilated by all vector fields tangent to the leaves of $\mathscr{F}$; it is easy to see that this is equivalent to the statement that $f$ and $\bar{f}$ are both CR. We will use the same criterion to pick out those distributions and hyperfunctions which are constant on leaves.

If $S$ is real-analytic then any holomorphic function $f$ on a component $M_{+}$ or $M_{-}$of $M \backslash S$ induces a CR hyperfunction $b_{ \pm}(f)$ on $S$ [PW, HM]; $b_{ \pm}(f)$ is thought of as representing the "boundary values" of $f$, and any CR hyperfunction $f$ may be represented locally as a "jump" $b_{+}\left(f_{+}\right)-b_{-}\left(f_{-}\right)$.

\section{Straightness}

We begin by considering the case of a real-analytic Levi-flat hypersurface $S$ in a complex manifold $M$. The induced foliation $\mathscr{F}$ of $S$ will also be realanalytic, so that $\mathscr{F}$ is given locally near a point $p \in M$ by the level sets of a realvalued analytic function $f$ with $d f(p) \neq 0$. The function $f$ certainly satisfies the tangential Cauchy-Riemann equations on $S$, so by a well-known argument [AF] $f$ extends to a holomorphic function $\tilde{f}$ defined on a neighborhood of $p$ in $M$. Thus the pluriharmonic function $r:=\operatorname{Im} \tilde{f}$ vanishes along $S$ with $d r(p) \neq 0$, so that $r$ is a defining function for $S$ near $p$. (A defining function $r$ for a hypersurface $S$ is a smooth real-valued function vanishing precisely on $S$ with $d r(p) \neq 0$ for all $p \in S$.) Choosing holomorphic coordinates near $p$ so that $\tilde{f}(z)=z_{1}$, we see that $S$ is locally of the form $\left\{z: \operatorname{Im} z_{1}=0\right\}$, while the leaves of $\mathscr{F}$ are given locally by $z_{1}=a, a \in \mathbb{R}$.

The function $f$ used in the preceding paragraph is not uniquely determined, but any two choices $f_{1}$ and $f_{2}$ must be functionally dependent near $p$; that is, $f_{2}=\phi \circ f_{1}$ near $p$ for some real-analytic $\phi$. But it follows that $\tilde{f}_{2}=\tilde{\phi} \circ \tilde{f}_{1}$ near $p$, where $\tilde{\phi}$ is the complex-analytic extension of $\phi$. Thus $\tilde{f}_{1}$ and $\tilde{f}_{2}$ have the same level sets near $p$; the construction globalizes to show that $\mathscr{F}$ extends to a codimension-one holomorphic foliation defined on a neighborhood of $S$ in $M$. (Compare [Re, BdB].) Note the the notion of Schwarz reflection extends naturally to yield an antiholomorphic self-map of the leaf space of $\mathscr{F}$; by looking at deformations of elliptic curves, for example, it is not hard to 
see that leaves corresponding under reflection need not in general be either biholomorphic or conjugate-biholomorphic to each other.

It is natural at this point to inquire as to whether or not $S$ admits a global pluriharmonic defining function $r$. We adopt the notation $d^{c}:=i(\bar{\partial}-\partial)=J d$, where $J$ denotes the complex structure tensor, so that $r$ is pluriharmonic if and only if the one-form $d^{c} r$ is closed. Suppose that $S$ does admit a pluriharmonic defining function $r$. Then $r$ is constant on each leaf of $\mathscr{F}$, so $\bar{\partial} r, \partial r$, and $d^{c} r$ vanish along leaves of $\mathscr{F}$. Also the condition $d r \neq 0$ along $S$ guarantees that the closed one-form $\eta:=\left.d^{c} r\right|_{S}$ is nonvanishing. The form $\eta$ is said to define the foliation $\mathscr{F}$.

We claim that conversely $S$ admits a global pluriharmonic defining function if $\mathscr{F}$ is defined by a nonvanishing closed real-analytic one-form $\eta$ on $S$. In fact, in this situation we can insist that the function $f$ used above satisfy $d f=\eta$. Any two such choices differ by a real additive constant, so that $r=\operatorname{Im} \tilde{f}$ gives a well-defined global pluriharmonic defining function for $S$. Thus we have proved the following.

Proposition 1. A real-analytic Levi-flat hypersurface $S$ admits a global pluriharmonic defining function if and only if the induced foliation $\mathscr{F}$ is defined by $a$ nonvanishing real-analytic closed one-form.

Note that the condition in Proposition 1 depends only on the real-analytic structure of the foliation. Note also that if a compact hypersurface $S$ does admit a global pluriharmonic defining function $r$ then any component $U$ of $M \backslash S$ contains a compact Levi-flat hypersurface $S_{\varepsilon}:=\{z \in U: r(z)=\varepsilon\}$ so that (as explained in the introduction) $U$ does not carry a strictly plurisubharmonic function.

We turn now to the case where $S$ is a Levi-flat hypersurface of class $C^{\infty}$. We shall say that $S$ is straight if for each $p \in S$ there are holomorphic local coordinates $\left(z_{1}, \ldots, z_{n}\right)$ on $M$ near $p$ so that $S$ admits a defining function near $p$ depending on $z_{1}$ alone. We have seen that $S$ is automatically straight in the analytic case. Rea [Re] showed that $S$ need not be straight in the $C^{\infty}$ case, and Bedford and deBartolomeis showed that $S$ may fail to be straight even in a suitable one-sided sense [BdB]. We note that if $S$ is straight then the foliation $\mathscr{F}$ extends to a neighborhood of $S$ in $M$ as before, and conversely the extendability of $\mathscr{F}$ implies the straightness of $S$ [BdB].

We note also that the proof of Proposition 1 can be adapted to the $C^{\infty}$ case to show that $S$ admits a defining function $r$ which satisfies the pluriharmonicity condition $d d^{c} r=0$ to infinite order along $S$ if and only if $\mathscr{F}$ is defined by a smooth nonvanishing closed one-form. We pose the following

Question. Does there exist a compact smooth Levi-flat hypersurface $S$ with Levifoliation $\mathscr{F}$ defined by a smooth nonvanishing closed one-form, such that some component of $M \backslash S$ carries a strictly plurisubharmonic function?

The following proposition about boundary values will be used in $\S 4$.

Proposition 2. Let $S$ be real-analytic and Levi-flat, and let $b(f)$ be a $C R$ hyperfunction induced by a holomorphic function $f$ on one side of $S$. If $b(f)$ is constant on leaves of $S$ then $f$ is constant on leaves of the extended foliation. 
Proof. Choose local coordinates so that $S=\left\{\operatorname{Im} z_{1}=0\right\}$. Then $\partial f / \partial z_{1}$ is holomorphic on one side of $S$, and $b\left(\partial f / \partial z_{1}\right)=\partial b(f) / \partial z_{1} \equiv 0$; hence $\partial f / \partial z_{1} \equiv 0$ by [PW]. Thus $f$ is constant on leaves in our local coordinate patch, and unique continuation gives the desired global result.

\section{INVARIANT LEVI-FLAT STRUCTURES ON $\mathbb{T}^{3}$}

Let $x_{1}, x_{2}, x_{3}$ be coordinates on $\mathbb{T}^{3}:=(\mathbb{R} / 2 \pi \mathbb{Z})^{3}$, and consider a complex vector field $L$ on $\mathbb{T}^{3}$ of the form

$$
L=\alpha\left(x_{3}\right) \frac{\partial}{\partial x_{1}}+\beta\left(x_{3}\right) \frac{\partial}{\partial x_{2}}+\gamma\left(x_{3}\right) \frac{\partial}{\partial x_{3}},
$$

where $\alpha, \beta$, and $\gamma$ are analytic functions of $x_{3}$. We will insist that

$$
\begin{gathered}
\operatorname{Im} \gamma \equiv 0, \quad \operatorname{Im} \alpha \equiv 1, \quad \operatorname{Im} \beta \equiv \mu, \quad \mu \text { constant, and } \\
\operatorname{Im} \alpha \bar{\beta} \text { never vanishes on } \mathbb{T} .
\end{gathered}
$$

These assumptions guarantee in particular that $L$ and $\bar{L}$ are everywhere linearly independent. $L$ is said to induce an (abstract) CR structure on $\mathbb{T}^{3}$, and the solutions of $L f=0$ are said to be CR functions/distributions/hyperfunctions. Furthermore, we have $[L, \bar{L}]=0$ so that the CR structure is automatically Levi-flat. The tangent space of the induced foliation $\mathscr{F}$ is spanned by $\operatorname{Re} L$ and $\operatorname{Im} L$, and the conformal structures on the leaves of $\mathscr{F}$ are determined by the condition that the restriction of $L$ to each leaf is a vector field of type $(0,1)$ along each leaf.

In a remark at the end of this section we will explore the relation between this class of examples and the more general class consisting of all $\mathbb{T}^{2}$-invariant real-analytic Levi-flat CR structures on $\mathbb{T}^{3}$.

Since the CR structure on $\mathbb{T}^{3}$ under consideration is real-analytic, there is an essentially unique complex manifold $M$ containing $S$ as a real-analytic hypersurface [AF]. In this case we can construct $M$ semiexplicitly as $\mathbb{T}^{3} \times$ $(-\varepsilon, \varepsilon)$ with complex structure given by the condition that the vector fields

$$
\widetilde{L}=\alpha(\zeta) \frac{\partial}{\partial x_{1}}+\beta(\zeta) \frac{\partial}{\partial x_{2}}+\gamma(\zeta) \frac{\partial}{\partial x_{3}}
$$

and

$$
X=\frac{1}{2}\left(\frac{\partial}{\partial x_{3}}+i \frac{\partial}{\partial t}\right)
$$

are of type $(0,1)$; here $\zeta=x_{3}+i t$, and $\alpha(\zeta), \beta(\zeta)$, and $\gamma(\zeta)$ are defined by complex-analytic extension. The vector fields $\widetilde{L}$ and $X$ together with their conjugates span the entire complexified tangent space of $M$, and $[\widetilde{L}, X]=0$, so the Newlander-Nirenberg Theorem (see for example [N]) guarantees that the above conditions uniquely determine a complex structure on $M$.

We are ready to state the following theorem.

Theorem 1. Let $S$ be the manifold $\mathbb{T}^{3}$ equipped with a Levi-flat $C R$ structure using a vector field $L$ as above, and let $\mathscr{F}$ and $M$ be the associated foliation and complexification. Then the following are equivalent:

(a) $S$ does not admit a global pluriharmonic defining function; 
(b) there is a neighborhood $U$ of $S$ in $M$ and a strictly plurisubharmonic function $\psi$ on $U \backslash S$ with $\psi(z) \rightarrow \infty$ as $z \rightarrow S$;

(c) $S$ admits $C R$ hyperfunctions which are not constant on leaves of $\mathscr{F}$;

(d) $\mathscr{F}$ contains a leaf with nontrivial holonomy;

(e) the orbits of the $\mathbb{T}^{2}$-action are neither always tangent nor always transverse to the leaves of $\mathscr{F}$;

(f) $\gamma$ has zeros on $\mathbb{T}$ but is not identically zero.

Remark. The notion of holonomy is explained in [CN, Chapter 4 and HH, III].

Proof. (e) $\Leftrightarrow(\mathrm{f})$ is trivial.

(d) $\Leftrightarrow$ (a) If $\mathscr{F}$ contains a leaf with nontrivial holonomy then by [HH, VIII, 1.1.2] $\mathscr{F}$ cannot be defined by a nonvanishing closed one-form; Proposition 1 now implies that (a) holds.

(b) $\Rightarrow$ (a) This follows from the maximum principle, as was explained in the introduction.

(a) $\Rightarrow$ (f) By Proposition 1 it suffices to show that the failure of (f) implies the existence of a nonvanishing real-analytic closed one-form $\eta$ defining $\mathscr{F}$.

Suppose that $\gamma \equiv 0$. Then we may take $\eta=d x_{3}$.

Suppose next that $\gamma$ has no zeros. Then we may take

$$
\eta=\mu d x_{1}-d x_{2}+\gamma^{-1}(\operatorname{Im} \alpha \bar{\beta}) d x_{3} ;
$$

$\eta$ is closed and vanishes on leaves by (3.2).

(f) and (b) $\Rightarrow$ (d) Suppose that $\gamma$ has a zero of finite order at $\zeta_{0}$. Then $l:=\left\{x \in \mathbb{T}^{3}: x_{3}=\zeta_{0}\right\}$ is a closed leaf of $\mathscr{F}$. If $l$ has trivial holonomy then all leaves of $\mathscr{F}$ coming near enough to $l$ are also closed [HH, III, 2.1]. The same must hold for the extended foliation constructed in $\S 2$; hence there are compact complex curves in $M$ arbitrarily close to $S$, contradicting (b).

(f) $\Leftrightarrow$ (c) We use the method of "separation of variables." Any hyperfunction $f$ on $S$ admits a Fourier expansion

$$
f\left(x_{1}, x_{2}, x_{3}\right)=\sum_{j, k \in \mathbb{Z}} f_{j k}\left(x_{3}\right) e^{i\left(j x_{1}+k x_{2}\right)} .
$$

We have $L f=0$ if and only if

$$
i(j \alpha+k \beta) f_{j k}+\gamma f_{j k}^{\prime}=0
$$

for all $j$ and $k$.

If $\gamma$ is identically zero then $f_{j k}$ must vanish unless $j \alpha+k \beta \equiv 0$, which by (3.2) implies that $j=k=0$. It follows that all CR hyperfunctions depend only on $x_{3}$ and thus are constant on leaves.

If $\gamma$ never vanishes then we must have

$$
f_{j k}=c_{j k} e^{-i \int \gamma^{-1}(j \alpha+k \beta) d x_{3}} ;
$$

periodicity forces $c_{j k}=0$ unless

$$
\frac{1}{2 \pi} \int_{0}^{2 \pi} \gamma^{-1}\left(x_{3}\right)\left(j \alpha\left(x_{3}\right)+k \beta\left(x_{3}\right)\right) d x_{3} \in \mathbb{Z} .
$$

By (3.2) this implies that $j+k \mu=0$, so that $f_{j k}$ also satisfies the conjugate version of (3.3) and thus $f$ is constant on leaves. 
Finally assume that $\gamma$ has $m$ zeros in a period interval, counting multiplicities. Then the hyperfunction theory of ordinary differential equations as developed in [Km2] tells us that the solution space on $\mathbb{R}$ of the differential equation (3.3) has dimension $m+1$, hence the space of periodic solutions has dimension at least $m$. On the other hand, using the conjugate version of (3.3) it is easy to show that a CR hyperfunction $f$ which is constant on leaves must have $f_{j k} \equiv 0$ unless $j+k \mu=0$. Hence there exist CR hyperfunctions which are not constant on leaves.

Remark. If $\gamma$ has multiple zeros then Komatsu's theory [Km1, Theorem 3.5] shows that (3.3) will have hyperfunction solution which are not distributions. Let us trace the consequences of this fact in an example. We let

$$
L=i \frac{\partial}{\partial x_{1}}+\frac{\partial}{\partial x_{2}}+\left(1-\cos x_{3}\right) \frac{\partial}{\partial x_{3}} \text {. }
$$

Then carrying out the computations outlined above we conclude that every CR hyperfunction admits an expansion

$$
\begin{aligned}
f\left(x_{1}, x_{2}, x_{3}\right)= & a \delta\left(x_{3}\right)+b \delta^{\prime}\left(x_{3}\right) \\
& +\sum_{(j, k) \neq(0,0)}\left\{a_{j k} e^{(j-i k) \cot \left(\frac{x_{3}+i 0}{2}\right)}+b_{j k} e^{(j-i k) \cot \left(\frac{x_{3}-i 0}{2}\right)}\right\} e^{i\left(j x_{1}+k x_{2}\right)} .
\end{aligned}
$$

If $f$ is a distribution then each term of the sum must also be a distribution; this forces $a_{j k}=0$ unless $j=0$ and $k<0$, and $b_{j k}=0$ unless $j=0$ and $k>0$. It follows easily that all CR distributions are constant on leaves.

Note also that

$$
\left(x_{1}, x_{2}, x_{3}, t\right) \mapsto\left(e^{i x_{1}+\cot (\zeta / 2)}, e^{i x_{2}-i \cot (\zeta / 2)}\right)
$$

is a biholomorphic map of a component of $M \backslash S$ onto the complement of a compact set in $D:=\mathbb{C}^{*} \times \Delta^{*}$, where $\Delta^{*}$ is the punctured unit disc; that is to say, $S$ can serve as a boundary for $D$. Let $A^{-\infty}$ denote the algebra of holomorphic functions on $D$ whose growth is majorized by a polynomial in the reciprocal of the distance to $S$. Then each $h \in A^{-\infty}(D)$ gives rise to a CR distribution $b(h)$ on $S[\mathrm{St}]$. The work above in combination with Proposition 2 shows that $H$ is a function of the $\Delta^{*}$-variable alone; this can also be checked by studying the power series expansion of $h$. Thus in particular the algebra $A^{-\infty}$ is not dense in the algebra $\mathscr{O}(D)$ of holomorphic functions on $D$.

(f) $\Rightarrow$ (b) It clearly suffices to construct $\psi$ on each side of $M$ separately; we will work on the side where $t>0$.

Let $\widetilde{M} \cong \mathbb{T}^{2} \times \mathbb{R} \times(-\varepsilon, \varepsilon)$ be the covering manifold of $M$ obtained by dropping the periodicity condition in the $x_{3}$-variable, and let $\widetilde{S}$ be the corresponding hypersurface. We let $\zeta=x_{3}+i t$ as before, and we let $F_{1}(\zeta)$ and $F_{2}(\zeta)$ be branches of $\int \gamma^{-1}(\zeta) \alpha(\zeta) d \zeta$ and $\int \gamma^{-1}(\zeta) \beta(\zeta) d \zeta$, respectively, defined for small positive values of $t$. For $j=1,2$ we let $\kappa_{j}$ be the value of the constant function $F_{j}(\zeta+2 \pi)-F_{j}(\zeta)$.

Lemma. Let $\psi$ be a smooth real-valued function defined on a component $\tilde{U}$ of $\widetilde{M} \backslash \widetilde{S}$. If $\psi$ is independent of $x_{1}$ and $x_{2}$ then $\psi$ is strictly plurisubharmonic if and only if it is locally a strictly convex function of $\operatorname{Im} F_{1}$ and $\operatorname{Im} F_{2}$.

Before proving the Lemma we proceed to use it to construct the desired function $\psi$. 
Let $\left\{\nu_{k}\right\}_{k \in \mathbb{Z}}$ be the distinct zeros of $\gamma$ on the real axis listed in order, and let $I_{k}$ be the interval $\left(\nu_{k}, \nu_{k+1}\right)$. The functions $F_{1}$ and $F_{2}$ extend holomorphically across each $I_{k}$ and $G:=\mu F_{1}-F_{2}$ has a constant imaginary part $\lambda_{k}$ on $I_{k}$; the $\lambda_{k}$ satisfy $\lambda_{k+n}=\lambda_{k}+\operatorname{Im} \kappa$, where $n$ is the number of distinct zeros in a period interval and $\kappa=\mu \kappa_{1}-\kappa_{2}$. Note also that $F_{j}$ admits an expansion near $\lambda_{k}$ of the form

$$
F_{j}(\zeta)=\tilde{a}_{j}+a_{j, 0} \log \left(\zeta-\nu_{k}\right)+\sum_{l \geq-m, l \neq 0} a_{j, l}\left(\zeta-\nu_{k}\right)^{l},
$$

where $m+1$ is the order of vanishing of $\gamma$ at $\nu_{k}$ and

$$
\alpha\left(\nu_{k}\right) a_{2,-m}=\beta\left(\nu_{k}\right) a_{1,-m} \neq 0 .
$$

Similarly, $G$ admits the expansion

$$
G(\zeta)=\tilde{b}+b_{0} \log \left(\zeta-\nu_{k}\right)+\sum_{l \geq-m, l \neq 0} b_{l}\left(\zeta-\nu_{k}\right)^{l}
$$

where each $b_{l}=\mu a_{1, l}-a_{2, l}$ is real and $\operatorname{Im} \tilde{b}=\lambda_{k}$. From (3.2) and (3.6) it follows that

$$
a_{1,-m} \text { and } a_{2,-m} \text { are } \mathbb{R} \text {-linearly independent and } b_{-m} \neq 0 \text {. }
$$

The function $\psi$ will be constructed as a sum of two functions $\psi_{1}$ and $\psi_{2}$ satisfying the following requirements:

(i) $\psi_{1}$ and $\psi_{2}$ will be smooth positive functions of $\zeta=x_{3}+$ it defined for small positive $t$;

(ii) $\psi_{1}$ and $\psi_{2}$ will be periodic of period $2 \pi$ in $x_{3}$;

(iii) $\psi_{1}$, respectively, $\psi_{2}$, will be locally representable as a convex, respectively, strictly convex, function of $\operatorname{Im} F_{1}$ and $\operatorname{Im} F_{2}$;

(iv) $\psi_{2}(\zeta) \rightarrow \infty$ as $\zeta$ approaches any point in $\mathbb{R} \backslash\left\{\nu_{k}: k \in \mathbb{Z}\right\}$, while $\psi_{1} \rightarrow \infty$ as $\zeta \rightarrow \nu_{k}$.

By periodicity it will suffice to define $\psi_{1}$ in a rectangle of the form $\left|x_{3}\right|<$ $\pi+\varepsilon, 0<t<\varepsilon$. The bottom side of this rectangle must contain a zero $\nu_{k}$ of the function $\gamma$. Condition (3.8) implies that

$$
\left(\operatorname{Im} F_{1}(\zeta)\right)^{2}+\left(\operatorname{Im} F_{2}(\zeta)\right)^{2} \rightarrow \infty \quad \text { as } \zeta \rightarrow \nu_{k} .
$$

Let $\Phi$ be a positive strictly convex function on $\mathbb{R}^{2}$ such that $\Phi\left(x_{1}, x_{2}\right)=$ $\left(x_{1}-\kappa_{1}\right)^{2}+\left(x_{2}-\kappa_{2}\right)^{2}$ for $\left(x_{1}, x_{2}\right)$ in some very large ball but $\Phi\left(x_{1}, x_{2}\right)=$ $x_{1}^{2}+x_{2}^{2}$ for $\left(x_{1}, x_{2}\right)$ near $\infty$. Then we can let

$$
\psi_{1}(\zeta)= \begin{cases}\left(\operatorname{Im} F_{1}(\zeta)\right)^{2}+\left(\operatorname{Im} F_{2}(\zeta)\right)^{2} & \text { for }-\pi-\varepsilon<x_{3} \leq \nu_{k}, 0<t<\varepsilon \\ \Phi\left(\operatorname{Im} F_{1}(\zeta), \operatorname{Im} f_{2}(\zeta)\right) & \text { for } \nu_{k} \leq x_{3}<\pi+\varepsilon, 0<t<\varepsilon\end{cases}
$$

The function $\psi_{2}$ will be given in a one-sided neighborhood of each interval $I_{k}$ by the formula $\psi_{2}(\zeta)=\left(\operatorname{Im} G(\zeta)-\lambda_{k}\right)^{-2}$; note that this formula is indeed meaningful on a one-sided neighborhood of $I_{k}$ (since $G^{\prime} \neq 0$ ) and that the periodicity condition is satisfied thus far. The difficulty consists of arranging a suitable transition for $\psi_{2}$ near each $\nu_{k}$.

Let us assume first that $\gamma$ has a simple zero at $\nu_{k}$ so that $\lambda_{k}=\lambda_{k-1}-\pi b_{0}$ and in particular $\lambda_{k} \neq \lambda_{k-1}$. The expansion (3.7) reveals that $\operatorname{Im} G(\zeta)$ lies between $\lambda_{k-1}$ and $\lambda_{k}$ as long as $\zeta$ is close enough to $\nu_{k}$. Let $\chi$ be a smooth 
positive convex function defined on the interval $\left(\lambda_{k-1}, \lambda_{k}\right)$ or $\left(\lambda_{k}, \lambda_{k-1}\right)$ with $\chi(s)=\left(s-\lambda_{j}\right)^{-2}$ for $s$ near $\lambda_{j}$. Then we may define $\psi_{2}$ near $\nu_{k}$ by the formula $\psi_{2}(\zeta)=\chi(\operatorname{Im} G(\zeta))$.

Now we treat the case where $\gamma$ has a zero of multiplicity $m>0$ at $\nu_{k}$. Using (3.7) and (3.8) and restricting our attention to a small enough neighborhood of $\nu_{k}$ we have

$$
\operatorname{Im} G(\zeta) \neq \lambda_{k} \quad \text { when } 0<\arg \left(\zeta-\lambda_{k}\right)<\pi / 2 m
$$

and similarly

$$
\operatorname{Im} G(\zeta) \neq \lambda_{k-1} \quad \text { when } \pi-\pi / 2 m<\arg \left(\zeta-\lambda_{k}\right)<\pi ;
$$

furthermore we may assume that $\operatorname{Im} G(\zeta)$ is very large in absolute value when $\arg \left(\zeta-\lambda_{k}\right)$ is close to $\pi / 2 m$ or $\pi-\pi / 2 m$. Pick positive locally convex functions $\chi_{1}$ and $\chi_{2}$ defined on $\mathbb{R} \backslash\left\{\lambda_{k-1}\right\}$ and $\mathbb{R} \backslash\left\{\lambda_{k}\right\}$, respectively, with $\chi_{1}(s)=$ $\left(s-\lambda_{k-1}\right)^{-2}$ for $s$ near $\lambda_{k-1}, \chi_{1}(s)=s^{2}$ for $s$ near $\pm \infty$, and correspondingly $\chi_{2}(s)=\left(s-\lambda_{k}\right)^{-2}$ for $s$ near $\lambda_{k}, \chi_{2}(s)=s^{2}$ for $s$ near $\pm \infty$. Then we may define $\psi_{2}$ near $\nu_{k}$ by setting

$$
\psi_{2}(\zeta)=\left\{\begin{aligned}
\chi_{1}(\operatorname{Im} G(\zeta)) & \text { when } \pi>\arg \left(\zeta-\lambda_{k}\right) \geq \pi-\pi / 2 m, \\
(\operatorname{Im} G(\zeta))^{2} & \text { when } \pi-\pi / 2 m \geq \arg \left(\zeta-\lambda_{k}\right) \geq \pi / 2 m, \\
\chi_{2}(\operatorname{Im} G(\zeta)) & \text { when } \pi / 2 m \geq \arg \left(\zeta-\lambda_{k}\right)>0
\end{aligned}\right.
$$

It is clear that periodicity of $\psi_{2}$ can be maintained in the patching processes described above, and the other required properties of $\psi_{1}$ and $\psi_{2}$ are transparent from the construction.

It remains to prove the Lemma.

Proof of Lemma. We may assume that $t>0$ on $\tilde{U}$.

Let

$$
h_{1}\left(x_{1}, x_{2}, x_{3}, t\right)=e^{i x_{1}-F_{1}(\zeta)}
$$

and

$$
h_{2}\left(x_{1}, x_{2}, x_{3}, t\right)=e^{i x_{2}-F_{2}(\zeta)}
$$

on $\tilde{U}$. It is easy to check that these two functions are holomorphic with independent differentials, so that $H=\left(h_{1}, h_{2}\right)$ is a holomorphic immersion of $\widetilde{U}$ into $\left(\mathbb{C}^{*}\right)^{2}$; if $\widetilde{U}$ is chosen to be rotation-invariant with respect to $x_{1}$ and $x_{2}$ then $H$ is $\mathbb{T}^{2}$-equivariant so that $\widetilde{U}$ is represented as a Reinhardt domain spread over $\left(\mathbb{C}^{*}\right)^{2}$. It follows now that $\psi$ is strictly plurisubharmonic if and only if it is locally a strictly convex function of $\log \left|h_{1}\right|=\operatorname{Im} F_{1}$ and $\log \left|h_{2}\right|=\operatorname{Im} F_{2}$.

Remark. The map $H$ does not induce a map from $U$ into $\left(\mathbb{C}^{*}\right)^{2}$ unless $\kappa_{1}$ and $\kappa_{2}$ lie in $2 \pi \mathbb{Z}$. Indeed we have

$$
h_{1}\left(x_{1}, x_{2}, x_{3}+2 \pi, t\right)=e^{-i \kappa_{1}} h_{1}\left(x_{1}, x_{2}, x_{3}, t\right)
$$

and

$$
h_{2}\left(x_{1}, x_{2}, x_{3}+2 \pi, t\right)=e^{-i \kappa_{2}} h_{1}\left(x_{1}, x_{2}, x_{3}, t\right) .
$$

Thus the "deck transformations" of $\widetilde{M}$ correspond to translations in logarithmic coordinate space. 
The arguments used above in the proof of (f) $\Leftrightarrow$ (c) show that for generic values of $\kappa_{1}$ and $\kappa_{2}$ the complex manifold $U$ admits no nonconstant holomorphic functions despite the existence of $\psi$. See [Ba] for related references.

This concludes the proof of Theorem 1.

Remark. It can be shown that an arbitrary $\mathbb{T}^{2}$-invariant real-analytic Levi-flat CR structures on $\mathbb{T}^{3}$ is given by a vector field as in (3.1) and furthermore that the normalizations (3.2) can be achieved (after an equivariant change of coordinates and multiplying $L$ by a complex scalar function) with the following exceptions:

(i) it may be necessary to pass to a double cover in the $x_{3}$-variable to arrange that $\operatorname{Im} \gamma \equiv 0$.

(ii) $\operatorname{Im} \alpha \bar{\beta}$ may have zeros on $\mathbb{T}$ (disjoint from the zeros of $\gamma$ ).

It is possible to modify the proof of Theorem 1 to show that conditions (a) through (f) are still equivalent.

\section{DisC BUNDLES OVER COMPACT RIEMANN SURFACES}

Another natural family of compact three-dimensional Levi-flat hypersurfaces consists of boundaries of (locally trivial) disc bundles over compact Riemann surfaces. Here we can get some results by slight modification of arguments of Diederich and Ohsawa, who have used the theory of harmonic mappings to study pseudoconvexity properties of disc bundles over compact Kähler manifolds.

We note that since the unit disc $\Delta$ admits no complex families of automorphisms, the transition functions for a disc bundle must be locally constant. A section of such a bundle is said to be flat if it is a locally constant function with respect to all local trivializations.

Note that every disc bundle is contained in a flat $\hat{\mathbb{C}}$-bundle constructed from the same transition functions. (Here $\hat{\mathbb{C}}$ denotes the Riemann sphere.)

Theorem 2. Let $\Omega \rightarrow X$ be a disc bundle over a compact Riemann surface $X$, let $M \rightarrow X$ be the associated $\hat{\mathbb{C}}$ bundle, and let $S$ be the boundary of $\Omega$ in $M$. Then conditions (a) through (d) of Theorem 1 are each equivalent to the following condition:

(g) the bundle $\Omega \rightarrow X$ does not admit a flat section.

Proof. ( $(\mathrm{g}) \Rightarrow$ (b) Diederich and Ohsawa show that the total space of any disc bundle over a compact Kähler manifold admits a smooth plurisubharmonic exhaustion function $\phi$. It suffices to modify $\phi$ to obtain an exhaustion function which is strictly plurisubharmonic near $S$ in the case where the base manifold is one-dimensional and ( $\mathrm{g}$ ) holds.

The key step in [DO] is Theorem 2 which affirms the existence of either a harmonic section of $\Omega \rightarrow X$ or a flat section of the circle bundle $S \rightarrow X$. The construction of $\phi$ is different in the two cases, and thus our slight modification will also divide into cases.

Case 1. We first examine the case where $\Omega \rightarrow X$ admits a harmonic section. With respect to a local trivialization this section is given by a map $h$ into the 
unit disc satisfying the nonlinear elliptic equation

$$
\partial \bar{\partial} h+\frac{2 \bar{h}}{1-h \bar{h}} \partial h \wedge \bar{\partial} h=0 .
$$

Diederich and Ohsawa set

$$
\phi(z, w)=-\log \left(1-\left|\frac{w-h(z)}{\bar{w} h(z)-1}\right|^{2}\right) .
$$

Then $\phi$ is plurisubharmonic and

$$
(\partial \bar{\partial})^{2} \phi=\left\{\nu \wedge \bar{\nu}+2|\xi(z, w)|^{2}\left(1-|\xi(z, w)|^{2}\right) \frac{\partial h \wedge \overline{\partial h}}{(1-h \bar{h})^{2}}\right\} \frac{d w \wedge d \bar{w}}{(1-w \bar{w})^{2}},
$$

where

$$
\xi(z, w)=\frac{\bar{w}-\overline{h(z)}}{1-\bar{w} h(z)} \quad \text { and } \quad \nu=\frac{\xi(z, w)^{2} \partial h-\partial \bar{h}}{1-h \bar{h}} .
$$

Thus away from the graph of $h, \partial \bar{\partial} \phi$ degenerates only when $\partial h=\bar{\partial} h=0$, i.e., only at critical points of $h$.

We claim that the critical points of $h$ are isolated. Let $z_{0}$ be such a critical point; by the Möbius-invariance of (4.1) we may assume that $h\left(z_{0}\right)=0$. Now by hypothesis $h$ is not constant and by the ellipticity of (4.1) $h$ is real-analytic [M]. Thus the Taylor series of $h$ has nonzero terms. But it is easy to check from (4.1) that the first nonzero nonharmonic term in this series is of strictly higher order than the first nonzero holomorphic term and the first nonzero antiholomorphic term, and from this observation it follows that the critical points are indeed isolated.

We must now alter the function $\phi$ near the isolated critical point $z_{0}$. Let $\chi$ be a smooth function supported near $z_{0}$ which is identically one in a small neighborhood of $z_{0}$. Let $\tilde{\phi}(z, w)=\phi(z, w)+\varepsilon \chi(z)\left(|z|^{2}+|w|^{2}\right)$. Then it is easy to check that for small $\varepsilon$ the function $\tilde{\phi}$ is strictly plurisubharmonic away from a neighborhood of the graph of $h$.

Remark. The above argument actually yields a strictly plurisubharmonic exhaustion function for all of $\Omega$ except in the case where $h$ is holomorphic, in which case the graph of $h$ is a compact one-dimensional variety in $\Omega$.

Case 2 . In the case where $S$ does admit a flat section, Diederich and Ohsawa view $M$ as a (not necessarily flat) affine bundle over $X$ with structure group $w \mapsto \tilde{w}=e^{i \theta} w+b(z)$. The flat section of $S$ corresponds to the infinity section of $X$, so that $\Omega$ is given locally by an inequality of the form

$$
\operatorname{Re}(\alpha(z) w+\beta(z))>0
$$

with $\alpha$ and $\beta$ holomorphic. The plurisubharmonic exhaustion function given by Diederich and Ohsawa is locally of the form

$$
|w-c(z)|^{2}-\log \frac{\operatorname{Re}(\alpha(z) w+\beta(z))}{|\alpha(z)|},
$$

where $c(z)$ is harmonic and satisfies the transformation law $\tilde{c}(z)=e^{i \theta} c(z)+$ $b(z)$. Here we will use instead the function

$$
\phi(z, w)=|w-c(z)|^{2}+\frac{|\alpha(z)|}{\operatorname{Re}(\alpha(z) w+\beta(z))}
$$


which is also a well-defined plurisubharmonic exhaustion function. Straightforward but somewhat tedious calculation reveals that

$$
\begin{aligned}
& (\partial \bar{\partial}) \phi=\partial \bar{c} \wedge \bar{\partial} c+\partial(w-c) \wedge \overline{\partial(w-c)} \\
& \quad+(4|\alpha| \operatorname{Re}(\alpha w+\beta))^{-1}\left(\partial \alpha-\frac{\alpha \partial(\alpha w+\beta)}{\operatorname{Re}(\alpha w+\beta)}\right) \wedge\left(\overline{\partial \alpha-\frac{\alpha \partial(\alpha w+\beta)}{\operatorname{Re}(\alpha w+\beta)}}\right) \\
& \quad+\left(4(\operatorname{Re}(\alpha w+\beta))^{3}\right)^{-1}|\alpha| \partial(\alpha w+\beta) \wedge \overline{\partial(\alpha w+\beta)} .
\end{aligned}
$$

Now if $c$ is not holomorphic then $\phi$ is strictly plurisubharmonic except (possibly) over the isolated points where $\bar{\partial} c=0$, and the patching argument above will complete the construction.

If $c$ is holomorphic then we introduce the trivializing coordinate $\hat{w}=w-$ $c(z)$, replacing $\beta(z)$ by $\hat{\beta}(z)=\beta(z)+\alpha(z) c(z)$. Examination of (4.2) reveals that $\phi$ is strictly plurisubharmonic except where $\partial \alpha=\partial \hat{\beta}=0$. If $\alpha$ and $\hat{\beta}$ are nonconstant then these points are isolated and we again finish by patching. If $\alpha$ and $\hat{\beta}$ are constant then dividing the local defining functions for $\Omega$ by $|\alpha|$ we obtain a global defining function $\operatorname{Re} e^{i \theta} \hat{w}+\operatorname{Re} \hat{\beta}>0$ so that $\Omega$ is a product, contradicting $(\mathrm{g})$.

$(\mathrm{g}) \Rightarrow$ (c) The preceding argument in fact shows that $\Omega$ is Stein, or at least blows down to a Stein analytic set. Hence in particular $\Omega$ admits holomorphic functions which are not constant on leaves of the extended foliation $\mathscr{F}$. Any such function has hyperfunction boundary values along $S$. Proposition 2 now implies that (c) holds.

(g) $\Rightarrow$ (d) Since $\Omega \rightarrow X$ is a flat bundle it is obtained from a representation $\rho$ of the fundamental group $\pi_{1}(X)$ into Aut $\Delta$. To show that (d) holds it will suffice to show that $\rho\left(\pi_{1}(X)\right)$ contains hyperbolic or parabolic elements. But if on the contrary $\rho\left(\pi_{1}(X)\right)$ consists solely of elliptic elements then the following proposition shows that the elements of $\rho\left(\pi_{1}(X)\right)$ have a common fixed point, contradicting $(\mathrm{g})$.

Proposition 3. If $G$ is a subgroup of $\operatorname{Aut}(\Delta)$ whose nontrivial elements are all elliptic then the elements of $G$ have a common fixed point in $\Delta$.

Proof of Proposition 3. Using the conformal equivalence of $\operatorname{Aut}(\Delta)$ with the upper half-plane we have

$$
\operatorname{Aut}(\Delta) \cong P S L(2, \mathbb{R})=\{A \in G L(2, \mathbb{R}): \operatorname{det} A=1\} / \pm I .
$$

Recall that $A$ represents a hyperbolic automorphism if and only if $|\operatorname{trace} A|>$ 2 .

Let $A \in S L(2, \mathbb{R})$ represent a nontrivial element of $G$. After conjugation we may assume that

with $\rho \notin \pi \mathbb{Z}$. Let

$$
A=R(\rho):=\left(\begin{array}{cc}
\cos \rho & \sin \rho \\
-\sin \rho & \cos \rho
\end{array}\right)
$$

$$
B=\left(\begin{array}{ll}
a & b \\
c & d
\end{array}\right)
$$

represent any other element of $G$. We must show that the automorphisms induced by $A$ and $B$ have the same fixed points; this is equivalent to showing that $B=R(\theta)$ for some $\theta \in \mathbb{R}$. 
Let $f(\theta)=\operatorname{trace} R(\theta) \cdot B \cdot R(-\theta) \cdot B^{-1}$. By inspection, $f(\theta)=\alpha+\beta \cos 2 \theta+$ $\gamma \sin 2 \theta$ for some $\alpha, \beta, \gamma \in \mathbb{R}$. Moreover

$$
\gamma=\frac{1}{2} f^{\prime}(0)=\frac{1}{2} \operatorname{trace}\left\{R^{\prime}(0)-B \cdot R^{\prime}(0) \cdot B^{-1}\right\}=0, \quad f(0)=2,
$$

and

$$
f(\pi / 2)=a^{2}+b^{2}+c^{2}+d^{2}=2+(a-d)^{2}+(b+c)^{2} .
$$

If $a=d$ and $b=-c$ then $B$ is of the required form $R(\theta)$; otherwise $f(\theta)>2$ for $\theta \notin \pi \mathbb{Z}$ so that $A B A^{-1} B^{-1}$ induces a hyperbolic automorphism, contrary to hypothesis.

(b) $\Rightarrow$ (a) and (d) $\Rightarrow$ (a) These are proved as before.

(a) $\Rightarrow$ (g) Assume to the contrary the $\Omega \rightarrow X$ does admit a flat section. Then we may choose local trivializations making this flat section into the zero section, thereby reducing the structure group of the bundle to the group of rotations $w \mapsto e^{i \theta} w$. Then $\log |w|$ is a pluriharmonic defining function for $S$.

(c) $\Rightarrow(\mathrm{g})$ As in the preceding paragraph, if on the contrary $\Omega \rightarrow X$ does admit a flat section the the structure group of the bundle can be reduced to rotations. It follows that there is a $\mathbb{T}$-action on $M$ given with respect to a distinguished local trivialization by the formula $e^{i \theta} \cdot(z, w)=\left(z, e^{i \theta} w\right)$. Thus any CR hyperfunction $u$ on $S$ admits a Fourier expansion of the form

$$
u\left(z, e^{i \theta}\right)=\sum_{n \in \mathbb{Z}} f_{n}(z) e^{i n \theta} \quad\left(f_{n} \text { holomorphic }\right)
$$

with respect to a distinguished local trivialization, where the absolute values of the functions $f_{n}$ are globally well defined. The maximum modulus principle implies that the $f_{n}$ are constant so that $\bar{u}$ is also CR and hence $u$ is constant on leaves.

This completes the proof of Theorem 2 .

\section{SUSPENSIONS OF ANALYTIC DIFFEOMORPHISMS OF THE CIRCLE}

The examples discussed in the preceding two sections fall neatly into two categories, according to whether or not the conditions (a) through (d) jointly hold or jointly fail. It is natural to search for some related dichotomy which would provide a useful classification of general compact (orientable, real-analytic) Leviflat CR manifolds. A first step in this direction would be to investigate the relations between conditions (a) through (d) in this more general context.

Now conditions (a) and (d) have a purely foliation-theoretic content (courtesy of Proposition 1). The proof in $\S 2$ that (d) implies (a) holds in general, but the reverse implication is known to fail. (See for example the proof of Theorem 3 below; but note also the weak positive result discussed in [HH, VIII.3.3.2].)

Similarly, the proof that (b) implies (a) is general, but using some ideas from dynamics we will show that the converse fails. The author does not know the status of the remaining implications. (See Remarks below.)

Theorem 3. There exist compact orientable real-analytic Levi-flat CR manifolds $M$ which satisfy condition (a) of Theorem 1 but fail to satisfy conditions (b) and $(\mathrm{d})$.

Our examples will be based on the theory of diffeomorphisms of the unit circle as treated in [A2 and $\mathrm{H}$ ]. 
Let $\rho: \mathbb{T} \rightarrow \mathbb{T}$ be a real-analytic orientation-preserving diffeomorphism of the unit circle. To $\rho$ we may associate a Levi-flat CR manifold $S=S_{\rho}$ by taking the quotient of the Levi-flat CR manifold $\mathbb{T} \times \mathbb{C}^{*}$ by the CR automorphism $(\zeta, w) \mapsto(\rho(\zeta), \tau w)$, where $\tau$ is any complex constant lying outside the unit circle. We can construct a complex neighborhood of $S$ by using the complexanalytic extension of $\rho$ to take the corresponding quotient of a suitably-chosen neighborhood of $\mathbb{T} \times \mathbb{C}^{*}$ in $\mathbb{C}^{*} \times \mathbb{C}^{*}$.

The leaves of the Levi-foliation $\mathscr{F}$ of $S$ are the images of the curves $\{\zeta\} \times \mathbb{C}^{*}$ in $\mathbb{T} \times \mathbb{C}^{*}$. If $\zeta$ is a periodic point of $\rho$ then the corresponding leaf $\mathscr{L}_{\zeta}$ is a torus; otherwise $\mathscr{L}_{\zeta}$ is biholomorphic to $\mathbb{C}^{*}$. Similar remarks apply to the extension of $\mathscr{F}$ to a holomorphic foliation on a complex neighborhood of $S$.

Proposition 4. If there is a sequence of periodic points $\zeta_{j}$ for $\rho$ with $\left|\zeta_{j}\right| \neq 1$ but $\left|\zeta_{j}\right| \rightarrow 1$ then the hypersurface $S$ fails to satisfy condition (b).

Proof. The existence of such a sequence of periodic points implies that for any neighborhood $U$ of $S$ there are (infinitely many) compact complex curves in $U \backslash S$.

Proposition 5. The hypersurface $S$ fails to satisfy condition (a) if and only if $\rho$ is conjugate to a rotation in the group $\operatorname{Diffeo}_{+}^{\omega}(\mathbb{T})$ of real-analytic orientationpreserving diffeomorphisms of the circle.

Proof. If $\rho=\sigma^{-1} R \sigma$ where $\sigma \in \operatorname{Diffeo}_{+}^{\omega}(\mathbb{T})$ and $R$ is a rotation then the pluriharmonic defining function $r(\zeta, w)=\log |\sigma(\zeta)|$ for $\mathbb{T} \times \mathbb{C}^{*}$ is invariant under the mapping $(\zeta, w) \mapsto(\rho(\zeta), \tau w)$ so that it passes down to a pluriharmonic defining function for $S$.

Conversely, if $S$ admits a pluriharmonic defining function then by Proposition $1 \mathscr{F}$ is defined by a nonvanishing real-valued real-analytic closed one-form $\eta$. The pull-back $\tilde{\eta}$ of $\eta$ to $\mathbb{T} \times \mathbb{C}^{*}$ must vanish on the leaves $\{\zeta\} \times \mathbb{C}^{*}$ so that $\tilde{\eta}=f(\zeta, w) d \zeta$. The fact that $d \tilde{\eta}=0$ now implies that $f$ is a function of $\zeta$ alone so that $\tilde{\eta}$ is the pull-back of a nonvanishing real-valued real-analytic one-form $\hat{\eta}$ on $\mathbb{T}$. The invariance of $\tilde{\eta}$ under the deck transformations implies that $\rho^{*} \hat{\eta}=\hat{\eta}$. By multiplying $\hat{\eta}$ be a real constant we may assume that $\int_{\mathbb{T}} \hat{\eta}=2 \pi$. Define $\sigma: \mathbb{T} \rightarrow \mathbb{T}$ by $\sigma(\zeta)=\exp \left(i \int_{1}^{\zeta} \hat{\eta}\right)$. Then $\sigma \in \operatorname{Diffeo}_{+}^{\omega}(\mathbb{T})$ and

$$
\sigma(\rho(\zeta))=\exp \left(i \int_{1}^{\rho(\zeta)} \hat{\eta}\right)=\exp \left(i \int_{\rho^{-1}(1)}^{\zeta} \hat{\eta}\right)=\exp \left(i \int_{\rho^{-1}(1)}^{1} \hat{\eta}\right) \cdot \sigma(\zeta)
$$

so that $\rho=\sigma^{-1} R \sigma$, where $R$ denotes rotation by the angle $\int_{\rho^{-1}(1)}^{1} \hat{\eta}$.

Proposition 6. The hypersurface $S$ fails to satisfy condition (d) if and only if $\rho$ is conjugate to a rotation in the group $\mathrm{Homeo}_{+}(\mathbb{T})$ of orientation-preserving homeomorphisms of the circle.

Proof. The leaf $\mathscr{L}_{\zeta_{0}}$ has nontrivial holonomy if and only if $\zeta_{0}$ is a boundary point of the closed set $P_{k}:=\left\{\zeta \in \mathbb{T}: \rho^{\circ k}(\zeta)=\zeta\right\}$ for some positive integer $k$. Thus $\mathscr{F}$ is without holonomy if and only if each $P_{k}=\varnothing$ or $\mathbb{T}$. But it follows easily from Denjoy's theorem (see (5.4) below) that the latter condition is equivalent to the conjugacy of $\rho$ in $\operatorname{Homeo}_{+}(\mathbb{T})$ to a rotation. 
Suppose that we can construct $\rho \in \operatorname{Diffeo}_{+}^{\omega}(\mathbb{T})$ such that $\rho$ is conjugate in $\mathrm{Homeo}_{+}(\mathbb{T})$ to an irrational rotation and $\rho$ has a sequence of periodic points accumulating on $\mathbb{T}$.

Then $\rho$ cannot be conjugate in $\operatorname{Diffeo}_{+}^{\omega}(\mathbb{T})$ to a rotation, for by extending the conjugating map homomorphically to a neighborhood of $\mathbb{T}$ it would follow easily that $\rho$ is periodic-point free in a neighborhood of $\mathbb{T}$. Thus the corresponding hypersurface $S$ would satisfy the specifications of Theorem 3 .

Now the existence of diffeomorphisms $\rho$ satisfying (5.1) is evident (at least implicitly) in the papers $[\mathrm{A} 1, \mathrm{H}]$ but for the convenience of the reader we provide a few details.

If $\rho \in \operatorname{Diffeo}_{+}^{\omega}(\mathbb{T})$ then $\mathbb{T} \rightarrow \mathbb{T}, z \mapsto \mu(z) / z$ has winding number zero so that we may write $\rho(z) / z=e^{i f(z)}$ where $f$ is real-valued and analytic on $\mathbb{T}$. We define the rotation number of $\rho$ to be

$$
\mu_{\rho}=\exp \left\{i \lim _{n \rightarrow \infty} \frac{f(z)+f(\rho(z))+\cdots+f\left(\rho^{\circ n}(z)\right)}{n}\right\} .
$$

The following facts are well known (see for instance [A2, Chapter $3, \S 11 . \mathrm{GHI}]$ ):

(5.2) The above limit exists and is independent of $z \in \mathbb{T}$ and the choice of $f$ above.

(5.3) $\mu_{\rho}^{k}=1$ if and only if $\rho$ has a fixed point of order $k$ on $\mathbb{T}$.

(5.4) (Denjoy's theorem) If $\mu_{\rho}$ is not a root of unity then $\rho$ is conjugate in $\mathrm{Homeo}_{+}(\mathbb{T})$ to rotation by $\mu_{\rho}$.

Let us now pick a Blaschke quotient $\rho_{0} \in \operatorname{Diffeo}_{+}^{\omega}(\mathbb{T})$ of degree $>1$; for example we may let $\rho_{0}$ be the map

$$
\zeta \mapsto \frac{\zeta(\zeta-\varepsilon)(1+\bar{\varepsilon} \zeta)}{(1-\bar{\varepsilon} \zeta)(\zeta+\varepsilon)}
$$

for $\varepsilon$ small.

For $\lambda \in \mathbb{T}$ let $R_{\lambda}$ denote the rotation $\zeta \mapsto \lambda \zeta$. We note that for any positive integer $k$ the rational function $\left(R_{\lambda} \circ \rho_{0}\right)^{\circ k}$ has degree $>1$ and thus cannot coincide with the identity mapping on $\mathbb{T}[\mathrm{H}$, III.3.4].

Let $h(\lambda)=\mu_{R_{\lambda} \circ \rho_{0}}$. Then $h$ is a continuous orientation-preserving mapping from $\mathbb{T}$ to $\mathbb{T}$ with winding number 1 [H, III.1]. It follows from (5.4) above that $h^{-1}(\mu)$ is a single point when $\mu$ is not a root of unity. However, when $\mu$ is a root of unity then $h^{-1}(\mu)$ is an arc of $\mathbb{T}$ [H, III.2.7].

Let $K$ be the Cantor set obtained by deleting the interiors of all the abovementioned arcs. Let

$$
E_{n}=\left\{\lambda \in K: R_{\lambda} \circ \rho_{0} \text { has a periodic orbit in the annulus } 1-n^{-1}<|z|<1\right\} .
$$

Each $E_{n}$ is open by Hurwitz' Theorem. We claim that each $E_{n}$ is dense in $K$. In fact, if $\lambda_{0}$ is an endpoint of one of the deleted arcs then $R_{\lambda_{0}} \circ \rho_{0}$ has a periodic point on $\mathbb{T}$. We can find $\lambda$ lying arbitrarily close to $\lambda_{0}$ such that $h(\lambda)$ is not a root of unity and thus $R_{\lambda} \circ \rho_{0}$ has periodic points lying close to $\mathbb{T}$ but not on $\mathbb{T}$. Since the periodic points of $R_{\lambda} \circ \rho_{0}$ lying off of $\mathbb{T}$ come in pairs symmetric with respect to $\mathbb{T}$ we find that $E_{n}$ is indeed dense in $K$. 
Thus $E:=\bigcap_{n} E_{n}$ is residual in $K$ (in the sense of Baire category) and in particular $E$ is uncountable. Picking $\lambda \in E$ so that $h(\lambda)$ is not a root of unity we find that $\rho:=R_{\lambda} \circ \rho_{0}$ satisfies the conditions in (5.1) and hence the proof of Theorem 3 is complete.

Remarks. Let us examine condition (c) of Theorem 1 on the hypersurfaces $S_{\rho}$. Arguing as in $\S 3$ we find that CR hyperfunctions on $S_{\rho}$ admit expansions

$$
f(\zeta, w)=\sum_{j \in \mathbb{Z}} f_{j}(\zeta) w^{j},
$$

where the $f_{j}$ are hyperfunctions on $\mathbb{T}$ satisfying

$$
f_{j} \circ \rho=\tau^{-j} f_{j}
$$

$f$ is constant on leaves if and only if $f_{j}=0$ for $j \neq 0$.

If $\rho$ is conjugate in $\operatorname{Diffeo}_{+}^{\omega}(\mathbb{T})$ to a rotation then a Fourier series argument shows that (c) fails. If the rotation number of $\rho$ is a root of unity but $\rho$ is not conjugate in $\operatorname{Diffeo}_{+}^{\omega}(\mathbb{T})$ to a rotation then in view of the constructions in $\S 3$ the author is inclined to guess that (c) holds. The author is unwilling to hazard a guess regarding the case of diffeomorphisms $\rho$ satisfying (5.1).

The author does not know of a useful necessary and sufficient condition on $\rho$ for $S_{\rho}$ to satisfy condition (b) of Theorem 1 .

\section{REFERENCES}

[A1] V. I. Arnold, Small denominators I, Izv. Akad. Nauk SSSR Ser. Mat. 25 (1961), 21-86; English transl., Transl. Amer. Math. Soc. 46 (1965), 213-284.

[A2] V. I. Arnold, Geometrical methods in the theory of ordinary differential equations (2nd ed.), Springer-Verlag, 1988.

[AF] A. Andreotti and G. A. Fredricks, Embeddability of real analytic Cauchy-Riemann manifolds, Ann. Scuola Norm. Sup. Pisa (4) 6 (1979), 285-304.

[Ba] D. Barrett, A remark on the global embedding problem for three-dimensional CR manifolds, Proc. Amer. Math. Soc. 102 (1988), 888-892.

[BdB] E. Bedford and P. deBartolomeis, Levi flat hypersurfaces which are not holomorphically flat, Proc. Amer. Math. Soc. 81 (1981), 575-578.

[CN] C. Camacho and A. Neto, Geometric theory of foliations, Birkhaüser, Boston, Mass., Basel, and Stuttgart, 1985.

[DO] K. Diederich and T. Ohsawa, Harmonic mappings and disc bundles over compact Kähler manifolds, Publ. Res. Inst. Math. Sci. Kyoto Univ. 21 (1985), 819-833.

[DF] K. Diederich and J. E. Fornæss, A smooth pseudoconvex domain without pseudoconvex exhaustion, Manuscripta Math. 39 (1982), 119-123.

[HH] G. Hector and U. Hirsch, Introduction to the geometry of foliations, Parts A and B, Vieweg, Braunschweig and Wiesbaden, 1981/83.

[H] M. Herman, Sur la conjugaison différentiable des defféomorphismes du cercle à des rotations, Publ. Math. Inst. Hautes Etudes Sci. 49 (1979), 5-234.

[HM] C. D. Hill and B. MacKichan, Hyperfunction cohomology classes and their boundary values, Ann. Scuola Norm. Sup. Pisa (4) 4 (1977), 577-597.

[Km1] H. Komatsu, An introduction to the theory of hyperfunctions, Lecture Notes in Math., vol. 287, Springer, 1973, pp. 30-40.

[Km2] H. Komatsu, On the index of ordinary differential operators, J. Fac. Sci. Univ. Tokyo Sect. 1 A 18 (1971), 379-398.

[M] C. Morrey, Multiple integrals and the calculus of variations, Springer, 1966. 
[N] L. Nirenberg, Lectures on linear partial differential equations, CBMS Regional Conf. Ser. in Math., no. 17, Amer. Math. Soc., Providence, R.I., 1973.

[Oh] T. Ohsawa, A Stein domain with smooth boundary which has a product structure, Publ. Res. Inst. Math. Sci. Kyoto Univ. 18 (1982), 1185-1186.

[PW] J. Polking and R. O. Wells, Jr., Boundary values of Dolbeault cohomology classes and a generalized Bochner-Hartogs theorem, Abh. Math. Sem. Univ. Hamburg 47 (1978), 3-24.

[Re] C. Rea, Levi-flat submanifolds and holomorphic extensions of foliations, Ann. Scuola Norm. Sup. Pisa Sci. Fis. Mat. (3) 26 (1972), 665-681.

[St] E. Straube, Harmonic and analytic functions admitting a distribution boundary value, Ann. Scuola Norm. Sup. Pisa Cl. Sci. (4) 10 (1984), 559-591.

Department of Mathematics, University of Michigan, Ann Arbor, Michigan 481091003 